\title{
Surface Reconstruction of Ancient Water Storage Systems An Approach for Sparse 3D Sonar Scans and Fused Stereo Images
}

\author{
Erik A. Nelson ${ }^{1}$, Ian T. Dunn ${ }^{1}$, Jeffrey Forrester ${ }^{1}$, \\ Timothy Gambin ${ }^{2}$, Christopher M. Clark ${ }^{3}$ and Zoë J. Wood ${ }^{1}$ \\ ${ }^{1}$ Computer Science Department, California Polytechnic State University, San Luis Obispo, CA, USA \\ ${ }^{2}$ Department of Classics and Archaeology, University of Malta, Msida, Malta \\ ${ }^{3}$ Department of Engineering, Harvey Mudd College, Claremont, CA, USA
}

Keywords: Geometric Reconstruction, Underwater Stereo Vision, Level Sets.

\begin{abstract}
This work presents a process pipeline that addresses the problem of reconstructing surfaces of underwater structures from stereo images and sonar scans collected with a micro-ROV on the islands of Malta and Gozo. Using a limited sensor load, sonar and small GoPro Hero2 cameras, the micro-ROV is able to explore water systems and gather data. As a preprocess to the reconstruction pipeline, a $3 \mathrm{D}$ evidence grid is created by mosaicing horizontal and vertical sonar scans. A volumetric representation is then constructed using a level set method. Fine-scale details from the scene are captured in stereo cameras, and are transformed into point clouds and projected into the volume. A raycasting technique is used to trim the volume in accordance with the projected point clouds, thus reintroducing fine details to the rough sonar-generated model. The resulting volume is surfaced, yielding a final mesh which can be viewed and interacted with for archaeological and educational purposes. Initial results from both steps of the reconstruction pipeline are presented and discussed.
\end{abstract}

\section{INTRODUCTION}

Many underwater sites such as cisterns, small sea caves, and other areas inaccessible to humans offer extraordinary opportunities for archaeological study. Remotely Operated Vehicles (ROVs) are commonly employed to explore such sites due to their small size, maneuverability, and sensor payload capacity. A common research goal between archaeologists and scientists exploring these sites is the ability to create accurate reconstructions of the geometry found within. These reconstructions can be used to visualize scale, structure, and water level, examine interesting features more closely, and potentially date the cisterns and surrounding sites.

In this work we focus on the creation of surface meshes of underwater cisterns and water galleries from a sparsely populated 3D evidence grid input (Fig. 1). The evidence grid input is created from a unified map of several horizontal and vertical sonar scans of walls and other geometry, which are collected with a sonar sensor mounted to a submersible micro-ROV (McVicker et al., 2012). Previous work has successfully reconstructed $2 \mathrm{D}$ and extruded 2.5D meshes of scanned surfaces using an iterative probabilistic hole filling approach and marching cubes, using 2D evidence grids from sonar scans as an input (Forrester et al., 2013). Unfortunately, this method does not generalize well to sparsely sampled 3D evidence grids, thus inhibiting the reconstruction of surfaces representative of true site geometry.

The work presented in this paper is motivated by an ongoing interdisciplinary project with the broad goal of exploring and mapping cisterns, water galleries, and shoreline caves on the islands of Malta, Gozo, and Sicily for archaeological study. These water storage cisterns, which date back as far as 3000

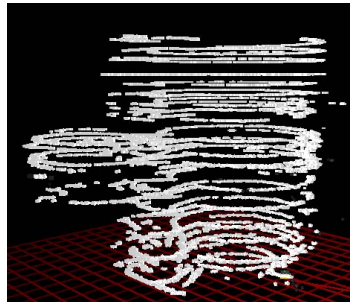

(a)

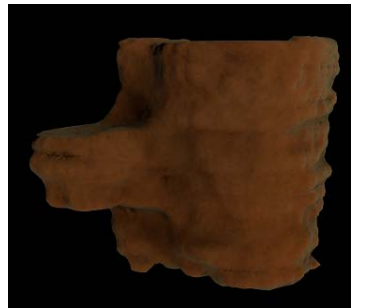

(b)
Figure 1: A two-chambered cistern in Mdina, Malta that was explored and mapped. (a) displays the evidence grid generated from 40 horizontal sonar scans taken while hovering the ROV up the cistern at $0.2 \mathrm{~m}$ intervals. (b) shows the water tight mesh produced by the first step of our pipeline. 


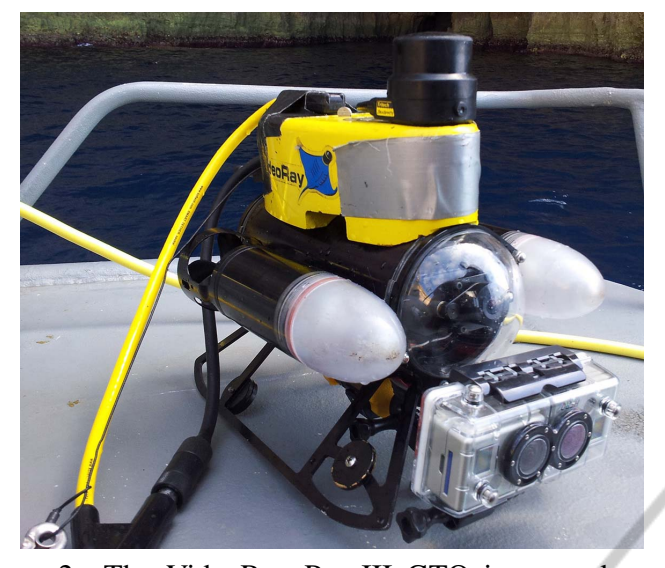

Figure 2: The VideoRay Pro III GTO is an underwater micro-ROV $(36.8 \mathrm{~cm} \times 28.9 \mathrm{~cm} \times 21.6 \mathrm{~cm})$, with a depth sensor, a compass, and a front and rear video camera. A removable Tritech Micron scanning sonar was mounted to the top and two vertically aligned GoPro Hero2 cameras were mounted to the front in a waterproof stereo casing.

B.C.E., were explored with a micro-ROV while collecting sonar scans, depth measurements, compass measurements, video and stereo images (Fig. 2). Six different expeditions have resulted in the exploration of over 100 sites. For more information on evidence grid generation and the ROV cistern mapping project, see (McVicker et al., 2012), (Forney et al., 2011), and (Dobke et al., 2013). Due to the small entryways to these water systems, limited sensors were used, making data collection challenging and resulting in fairly sparse sonar data and poor stereo images (albeit densely sampled). Our reconstruction pipeline must handle data with both: varying density and overall sparsity.

We use a level set algorithm for surface reconstruction of sparse 3D sonar data. Unlike other hole filling algorithms, level set methods are capable of producing closed surfaces regardless of the sparsity of the original evidence grid. The level set method outputs a 3D implicit surface which is used to compute a volume. The reconstructed volume serves as a good rough approximation of the shape of the site's true geometry.

To add finer geometric details, stereo images of interesting features captured within the cisterns are turned into point clouds and projected into the volume. The point cloud data is considerably denser in the local region it represents compared to the sparse 3D sonar data. To address this difference in density, the projected stereo point clouds are used as a base for raycasting, where all voxels in the volume lying beyond the projected point cloud have their occupancies set greater than zero. After trimming the volume, the newly introduced zero-crossings allow sur- facing algorithms such as marching cubes (Lorensen and Cline, 1987) to reintroduce stereo features previously omitted from the model. An overview of the algorithm pipeline is shown in Fig. 3.

Presented in this paper are the details behind applying the new reconstruction algorithm to $3 \mathrm{D}$ sonar and stereo image data. The proposed algorithm can produce water tight geometric models, representing complex underwater storage systems, even given sparse input data. We present results of three general surface reconstructions from sparse $3 \mathrm{D}$ sonar and one surface reconstruction with detailed geometry added via stereo imagery.

\section{RELATED WORKS}

Surface Reconstruction: Surface reconstruction of unorganized points in three dimensions is a well studied problem with many valid methods. One of the most popular recent approaches is Poisson Surface Reconstruction (Kazhdan et al., 2006). This method takes a point cloud with oriented point normals as its input and creates an indicator function (an inside outside table) which it can then use to determine connectivity of input points and extract a 3D model. Other closely related works include (Mullen et al., 2010) and work by Deng in (Deng et al., 2011) which use a variational approach, but either depend on denser samples or completely closed contours as input.

In contrast, level set methods (Zhao et al., 2001) take surface patches, curves and points as input and generate a distance function to input data. Based on the distance function created, an initial surface surrounding the input data is generated. The initial surface is updated based on vector and scalar fields generated from the moving surface interface and the input data. A final surface representative of the initial data set can be extracted when the initial surface reaches the input data. Given the sparsity of our input data, including partial contours and a lack of oriented normals, level set methods are more appropriate for creating rough starting models.

Underwater Stereo Reconstruction: The creation of accurate reconstructions from underwater stereo is a field of ongoing study. Stereo matching is a difficult process, complicated further by the underwater setting where non-uniform illumination, visibility falloff, and optical aberrations cripple matching algorithms that work well in air. Entire research endeavors have been devoted to characterizing attenuation and light transmittance through water as a function 


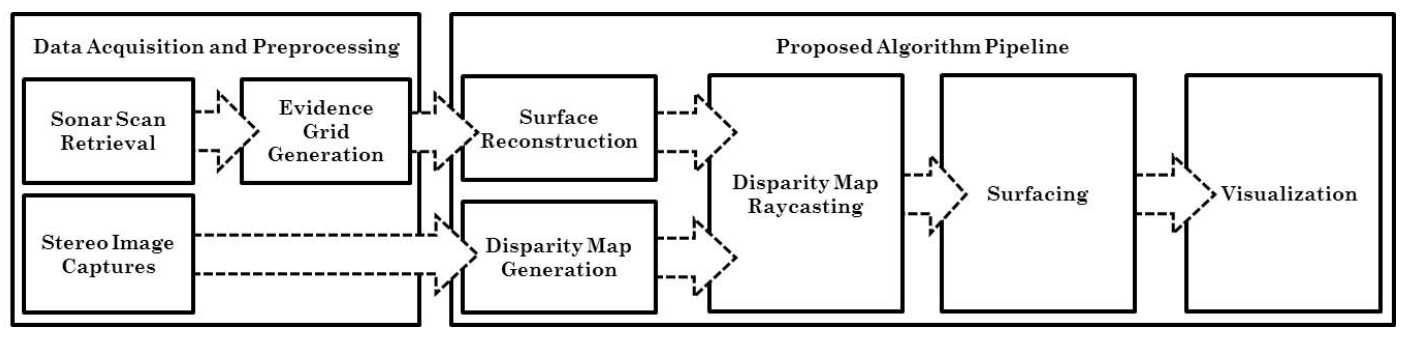

Figure 3: The proposed algorithm pipeline to create 3D reconstructions of cisterns.

of sediment levels and object distance for stereo purposes (Nascimento et al., 2009). In (Swirski et al., 2010), researchers were able to produce accurate disparity maps of underwater scenes using light flicker. However, there are no such light flicker effects in the underground cisterns explored in this project with which to base a stereo correspondence algorithm.

The same budget stereo camera system used in this project was utilized in (Schmidt and Rzhanov, 2012) to generate disparity maps of underwater scenes, resolving salient features to $\pm 3 \mathrm{~mm}$. However, the authors remarked that the cameras were not ideal due to their short $3.5 \mathrm{~cm}$ baseline.

Several research efforts have focused on reconstruction of underwater scenes from sensor information. For example, in (Beall et al., 2010) and (Drap et al., 2007), areas of the seafloor were reconstructed through image mosaicing. In (Hurtós et al., 2009), a sensor fusion approach is used to generate 3D mosaics of underwater settings using cameras, sonar, and other sensors on an AUV. Finally, in (Mahon et al., 2011), divers collected stereo images of a submerged town which were used to reconstruct a surface of the landscape. Campos (Campos et al., 2011) compares various surface reconstruction methods for sea floor data and the ARROV project (Papaleo and Puppo, 2004) includes reconstructions from sparse data from ROVs. While many of these projects provide good means of reconstructing underwater surfaces, few attempts have been made to model closed 3D manmade chambers with a micro-ROV and limited sensors without human aid. In addition, few of the available stereo matching algorithms account for poorly-lit underwater scenes with no sunlight penetration.

\section{SURFACE RECONSTRUCTION}

Due to the small entryways to the water systems we wish to model, only limited sensors can be used, (two GoPro HD Hero2 cameras and a Tritech SeaSprite sonar sensor). These limited sensors result in fairly sparse sonar data and poor stereo images. In order to construct the best representation of the underwa- ter system, our reconstruction pipeline must handle sparse data with varying density.

For our geometric reconstruction we take a 3D evidence grid obtained from a Video Ray Pro III GTO ROV and Tritech Micron scanning sonar (McVicker et al., 2012). Unlike previous surface reconstruction attempts of cisterns which were made from 2D data (Forrester et al., 2013), this work uses new 3D sonar data sets. The input $3 \mathrm{D}$ evidence grid data structure is a uniform grid with each cell containing a probability that the cell represents a solid surface (i.e. walls, stones, etc.). In order to turn this into a true point cloud we only accept cells having a probability greater than a threshold as points in the cloud. We wish to fit a surface to this input data that best represents the measured environment. Given the sparsity of the data, we use a level set method to fit a minimal surface the input points.

\subsection{Level Set Method}

Level set surface reconstruction works by starting with an initial surface that is a bounding volume of the input data and iteratively moving the surface towards the input data. This surface is represented as $\Gamma$, the zero level set of a function, $\phi$, in 3 dimensions.

In our implementation, we use a gradient flow model to move the surface, as described in (Zhao et al., 2001). Our adopted movement equation is:

$$
\Delta \phi=\Delta t|\vec{n}| d^{(P-1)} \vec{g} \cdot \vec{n}+\frac{1}{P} d \kappa
$$

where $\Delta t$ is a fixed time step, $\vec{n}$ is the gradient of $\phi, d$ is the distance to the nearest original data point, $\vec{g}$ is the gradient of the distance function, and $\kappa$ is the curvature of the current surface. $\vec{n}, \phi, d, \vec{g}$, and $\kappa$ are all functions of 3 dimensions represented by voxels in a volume. Eq. 1 is used to iteratively move the function $\phi$ so that its zero level set $\phi$ moves towards the final reconstructed surface.

During each iteration we update the values of $\phi, \vec{n}$, and $\kappa$ for the voxels in the volume. To increase performance, we only calculate these values for points in 
the narrow band, as described in (Adalsteinsson and Sethian, 1994).

We calculate $\phi$ to be the distance from each voxel to $\Gamma$. To calculate $\kappa$, we use the following equations, adopted from (Osher and Fedkiw, 2003):

$$
\begin{array}{r}
\kappa=\phi_{x}^{2} \phi_{y y}-2 \phi_{x} \phi_{y} \phi_{x y}+\phi_{y}^{2} \phi_{x x}+ \\
\phi_{x}^{2} \phi_{z z}-2 \phi_{x} \phi_{z} \phi_{x z}+\phi_{z}^{2} \phi_{x x}+ \\
\phi_{y}^{2} \phi_{z z}-2 \phi_{y} \phi_{z} \phi_{y z}+\phi_{z}^{2} \phi_{y y}
\end{array}
$$

Here, $\phi_{x}$ is the first partial derivative of $\phi$ in the $x$ direction. We use the second-order accurate central and finite difference formulae given in Eqs. (3)-(5).

$$
\begin{aligned}
\phi_{x} & =\frac{\phi_{i+1}-\phi_{i-1}}{2 \Delta x} \\
\phi_{x x} & =\frac{\phi_{i+1}-2 \phi_{i}+\phi_{i-1}}{\Delta x^{2}} \\
\phi_{x y} & =\left(\frac{\phi_{i+1}-\phi_{i-1}}{2 \Delta x}\right)\left(\frac{\phi_{i+1}-\phi_{i-1}}{2 \Delta x}\right)
\end{aligned}
$$

where $\phi_{y}, \phi_{z}, \phi_{y y}, \phi_{z z}, \phi_{x z}$, and $\phi_{y z}$ are similarly calculated.

$d$ must be calculated for all voxels in the volume, which can be a prohibitively large number of calculations. To efficiently calculate the distance function we use the fast marching method described in (Sethian, 2001).

Under ideal circumstances the surface reconstruction process can be exited once the surface no longer changes significantly between iterations - at this point a minimum surface of the input data has been reached. However, in sparse data sets such as those we collected from cisterns, the surface may be pulled through gaps in the data where a surface actually existed. We therefore allow for human input to end the surface reconstruction process early when an acceptable surface has been reached but before that surface has been pulled too far through holes in the data.

\section{STEREO RECONSTRUCTION}

While the 3D volumetric reconstructions from Sec. 3 give a broad idea of the true shape of the cistern geometry, the Tritech Micron scanning sonar fails to capture small features such as crevices, rocky walls, and archways due to a large $35^{\circ}$ vertical beam angle. In addition, both hardware and software resolution constraints are introduced in the scan retrieval and evidence grid generation preprocessing stages. These limitations cause the volumetric 3D reconstructions to omit many important small features. In the disparity merging step of our algorithm we account for the limited resolution in our volumetric reconstructions by reintroducing finer details captured in stereo images to the model. Note that stereo image pairs will be of a significantly higher resolution than the sparse $3 \mathrm{D}$ sonar grid in a local region. This difference in resolution of data is handled by our algorithm using a projective raycasting technique.

\subsection{Disparity Map Generation}

Fine-scale features from deployments into cisterns and caves are captured and stored in stereo image pairs using two vertically aligned GoPro Hero2 cameras. The captured stereo images have large barrel distortions due to the domed camera lenses and nonuniform illumination due to the ROV's poor ability to fully light the scene, so all stereo images are retouched by applying a constant lens and lighting correction. Stereo image pairs are then matched to create disparity maps using MATLAB's Computer Vision System toolbox. The resulting collection of disparity maps of interesting features are converted to points clouds, and projected into the volumetric reconstruction made in Sec. 3 through a 3D affine transform.

Stereo vision is inherently challenged by the underwater setting, and even after correction many stereo images were plagued with non-uniform illumination, visibility falloff, and optical aberrations. These complications led to difficulties in feature recognition and matching, and limited the quality and number of usable disparity maps.

\subsection{Disparity Map Raycasting}

Disparity maps are converted to $3 \mathrm{D}$ point clouds for raycasting, following:

$$
\mathbf{p}=\left[\begin{array}{c}
p_{x} \\
p_{y} \\
p_{z}
\end{array}\right]=\left[\begin{array}{c}
I_{x} \\
I_{y} \\
\delta\left(I_{x, y}\right)
\end{array}\right]
$$

where $\mathbf{p} \in \mathbf{P}$ is a point in the point cloud $\mathbf{P}, I$ is a disparity map, and $\delta\left(I_{x, y}\right)$ is the depth value associated in the intensity of pixel $I_{x, y}$ (Fig. 4). The $\delta$ function maps a disparity value between left and right stereo images to a true depth value. $\delta$ is formulated such that one unit in world-space corresponds to one meter in the real world. Point clouds are then individually assigned to projectors, which are implemented as user-controlled objects that may be manually rotated and translated within our program based on mouse and keyboard input to allow the user to align the features captured in the stereo images with features in 


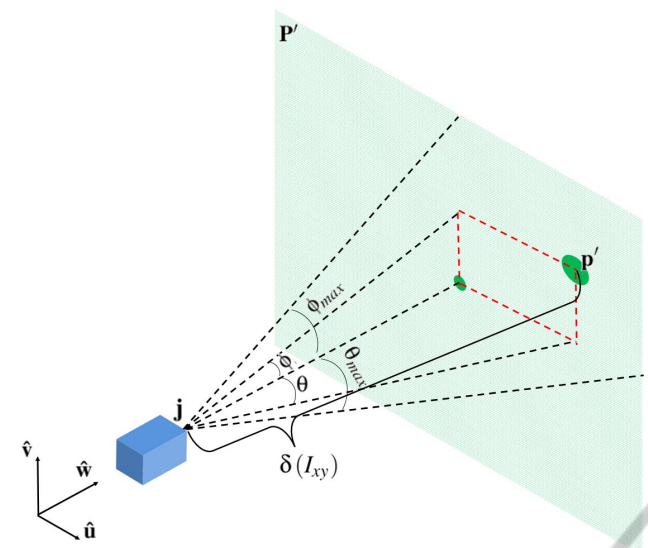

Figure 4: Projector and modified point cloud geometry.

the model. Rather than using the pixel space point cloud, $\mathbf{P}$, for raycasting, we project points outwards from each projector, $\hat{\mathbf{j}}$, in such a way that points are constrained within the projector frustum. This projection produces a new point cloud, $\mathbf{P}^{\prime}$.

$$
\begin{aligned}
\Xi \overline{\mathbf{P}^{\prime}} & =\underset{\theta \in \Theta}{\forall} \underset{\phi \in \Phi}{\forall} \mathbf{p}^{\prime}(\bar{\theta}, \phi) \\
\mathbf{p}^{\prime}(\theta, \phi) & =\mathbf{j}+\delta\left(I_{x, y}\right)(\hat{\mathbf{w}}+\sin (\phi) \hat{\mathbf{v}}+\alpha \sin (\theta) \hat{\mathbf{u}})
\end{aligned}
$$

where $\theta$ and $\phi$ are a horizontal and vertical angle along the projected image plane such that $\Theta=$ $\left\{-\theta_{\max }, \ldots, \theta_{\max }\right\}, \Phi=\left\{-\phi_{\max }, \ldots, \phi_{\max }\right\}$, and $\theta_{\max }$ and $\phi_{\max }$ equal half of the horizontal and vertical field of view of the GoPro Hero2 GTO cameras, $\alpha$ is the disparity map's aspect ratio, and $\hat{\mathbf{w}}, \hat{\mathbf{u}}$, and $\hat{\mathbf{v}}$ are the basis vectors of the projector.

Projectors are manually aligned in the volume to coincide with the observed locations of the real geometry captured in each point cloud. To aid the user in projector alignment, a marching cubes mesh is visualized within the volume so that the user may align projections with respect to the mesh itself.

Once projectors are situated, rays are cast through the viewports of the projectors. Rays originate at the projector and are cast through each point in $\mathbf{P}^{\prime}$. Bresenham's line algorithm (Bresenham, 1965) is continuously executed along each cast ray to find the next voxel in the ray's path. When the ray arrives at a voxel containing a point, a boolean switch is triggered, setting all following voxel occupancies greater than zero (denoting that the voxel is outside of the surface), altering the position of the eventual surface by redefining several zero-crossings along voxel edges (Fig. 5). Once a ray passes through a voxel whose occupancy is already greater than zero, the ray is terminated to assure that no surfaces are trimmed unintentionally.

While ray casting cannot guarantee that all voxels beyond the projected point cloud will be modified, the

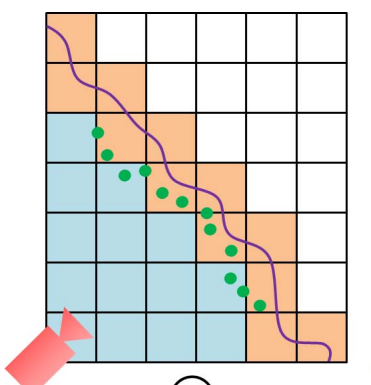

(1)

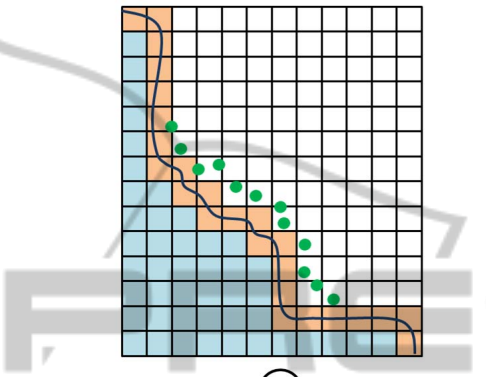

(3)

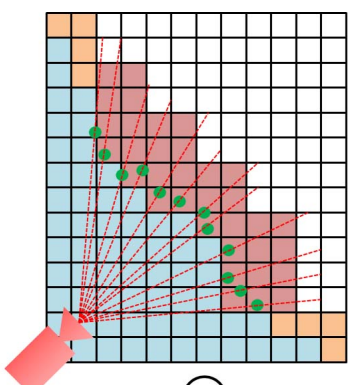

(2)

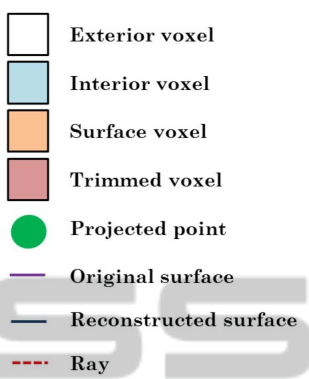

Figure 5: Point clouds are manually aligned near existing zero-crossings (orange to white boundary) (1). The volume is subdivided to provide finer resolution, and rays are cast from the projector through points (2). Voxels that lie beyond the intersected points have their occupancies set greater than zero, forming new zero-crossings for surfacing (3).

alterations to the volume occupancies generally occur near walls, so cast rays do not diverge far enough to miss any voxels. Additionally, projected point clouds may be sampled with sub-pixel accuracy, effectively minimizing the possibility of missed voxels.

The original resolution of a volume is decided based on the properties of the sonar sensor and the capabilities of the occupancy grid generation algorithm. Since the original resolution of the volume is only good enough to retain the details of the general surface, the volume is subdivided prior to raycasting to increase the amount of detail achieved in the areas which will be be modified by stereo data. In most cases the volume can be subdivided one to three times, yielding $8 \mathrm{x}$ to $512 \mathrm{x}$ as fine of a resolution. In order to facilitate smooth surface generation using marching cubes, voxel occupancies are interpolated trilinearly between subdivisions.

The algorithm is currently limited by memory consumption. Even with efficient data storage structures, when large volumes are subdivided in excess, they can exceed local memory. Due to the memory limitations, the current algorithm cannot truly add the same level of detail to the mesh as what is stored in the stereo images. Future work includes multi-resolution approaches to address this issue. 


\section{VISUALIZATION}

Marching cubes is run on the trimmed volume to produce a closed surface mesh, which can be visualized and interacted with by researchers. In addition to being able to manipulate the mesh in our visualization software, the interpolated marching cubes mesh is rendered in Cinema4D with a bump map and Fresnel shader to produce visually appealing static images and flyby videos.

Our visualization software also grants the ability to view errors in the level set reconstruction step by using a signed distance function to color map error onto vertices in the mesh. This visualization mode allows archaeologists to understand which areas of the reconstruction are likely to be most accurate.

\section{RESULTS}

The level set reconstruction method was applied to three data sets, including a mushroom shaped chamber (labeled "The Mush-room") from a large water gallery in Valletta, Malta, a complete water system connected by two ROV deployment entrances (labeled "Site 3+4") in Tal Gruwa, Gozo, and a complete two-chambered cistern (labeled "The Archives") in Mdina, Malta. The stereo reconstruction method was applied to The Mush-room.

Both horizontal and vertical sonar scans were collected in The Mush-room. During ROV deployment, the ROV was flown to a resting position, and two orthogonal $360^{\circ}$ sonar scans were collected. For more information on the double sonar scanning configuration see (Dobke et al., 2013). The collection of paired scans was fused into an evidence grid. Data for The Archives and Site $3+4$ was collected by horizontally scanning the cistern walls at 0.2 and 0.5 meter vertical intervals, respectively (with no vertical scans, due to hardware constraints). In some cases, scans would be duplicated and translated to a different depth in the evidence grid due to vertical symmetry in the walls of the cistern. This is demonstrated in the three planes of horizontal scans making up the lower channel in The Mush-room's evidence grid, and in some sonar scans present in Site 3+4. Note however, that these are all true 3D data sets as opposed to a single extruded horizontal layer as in (Forrester et al., 2013). The evidence grids for these three sites were processed into meshes and visualized (Fig. 6).

The level set technique described in Sec. 3 worked well in most situations, but caused ceilings to cave in in regions where gaps existed in the data. For example, the roof caved in on the Site $3+4$ mesh due to a

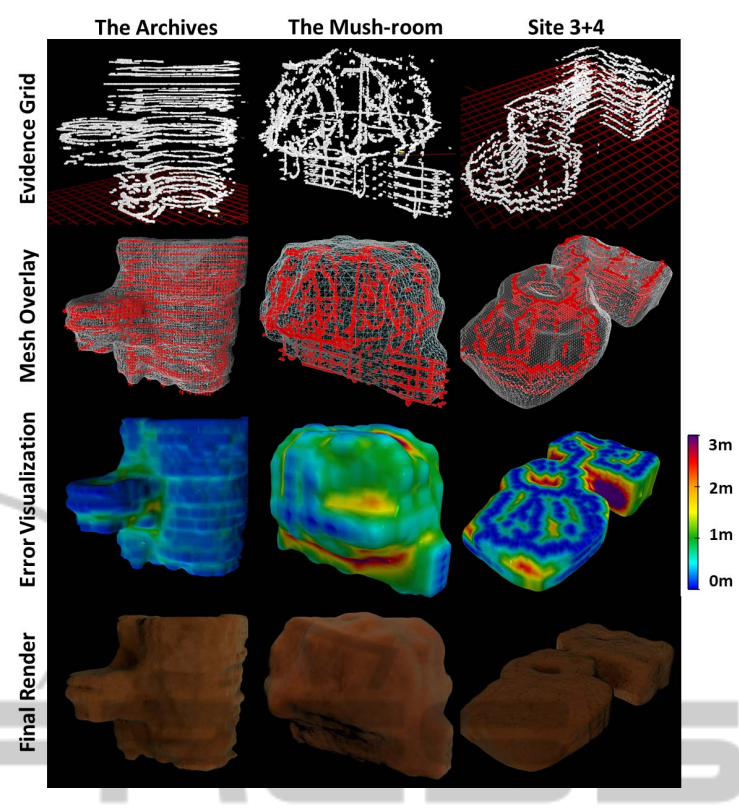

Figure 6: Results from applying the proposed 3D reconstruction technique to three large data sets. The first column displays the sparsely populated evidence grid input. The second column shows the reconstructed mesh overlaid on the input. The third column shows the reconstructed mesh colorized according to error in distance from the input points. The final column displays Cinema4D renders of the meshes given to archaeologists studying the sites.

lack of data in a circular central chamber. While these dimples were a source of error for data sets consisting of horizontal sonar scans, they were not present in models where vertical sonar scans were included in the evidence grid, such as The Mush-room.

Stereo images captured in The Mush-room were processed into disparity maps and used to trim the volume before resurfacing. Due to the poor quality of the GoPro Hero2 lenses and ROV lights, as well as the cloudiness of the water and plainness of features, it was extremely difficult to produce highquality disparity maps. Several methods, including prepackaged frameworks such as OpenCV and MATLAB's CV Toolbox, as well as three custom algorithms from stereo literature (Zitnick and Kanade, 2000) (Scharstein and Szeliski, 1998) (Nalpantidis and Gasteratos, 2010) were utilized in attempts to make good disparity maps. However, the distortions from the hardware as well as the plainness of the walls limited successful identification and matching of salient features. The disparity maps from MATLAB's CV Toolbox were used. To demonstrate the results of our algorithm, disparity maps were cleaned and mirrored in some situations. The initial stereo reconstruction results were not given to archaeologists 

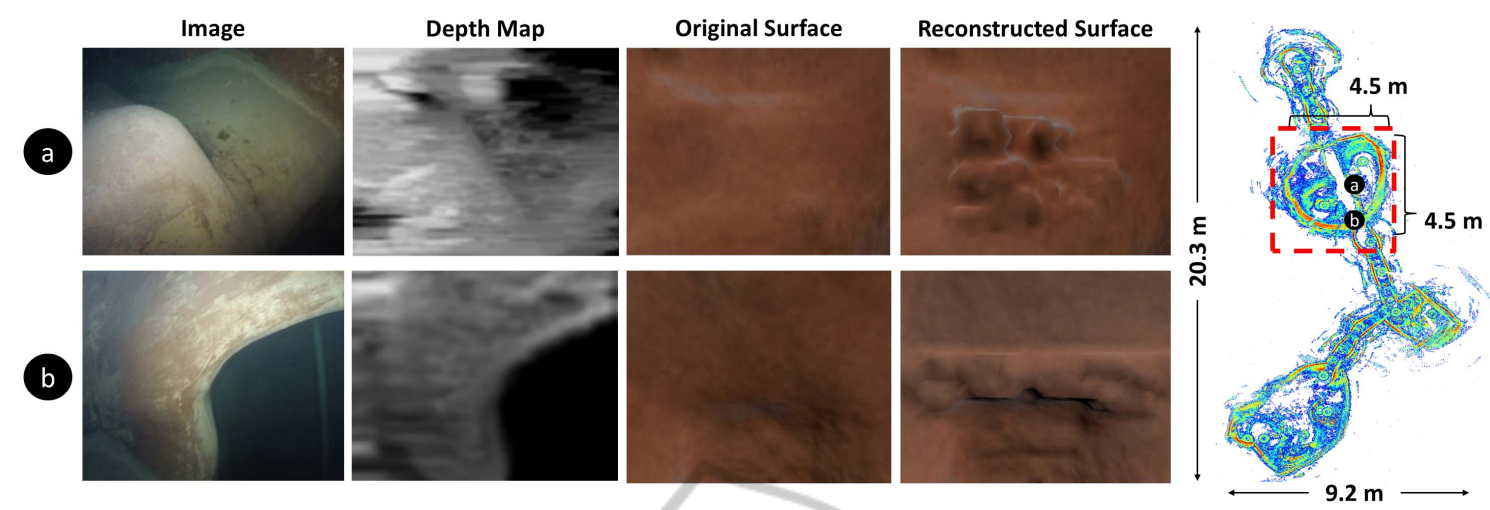

Figure 7: A sonar mosaic of the large water system containing The Mush-room (boxed in red). An archway (a) and a bump (b), which were omitted from the sonar model, were captured with stereo cameras within the cistern and converted to disparity maps. Due to the poor image quality feature matching was extremely difficult, and disparity maps were blurred and mirrored before being projected into the volume. The $4.5 \times 4.5 \times 5.2$ meter chamber was subdivided once to produce a $86 \times 68 \times 66$ voxel volume before raycasting (using an evidence grid cell size of 0.25 meters per voxel).

for study due to their variation from true geometry data, but are presented here as a proof of concept.

Two distinct features in The Mush-room, an archway and a large bump, were selected to add to the volume. The archway disparity map was mirrored to capture the entire feature, and both features were trimmed from the volume. Even in the presence of poor disparity maps, small features were reintroduced successfully (Fig. 7).

\section{CONCLUSIONS}

This work has presented a process pipeline that addresses the problem of reconstructing geometric models from 3D sonar scans of underwater settings with a micro-ROV. Surface reconstructions of underwater settings inaccessible to humans were produced for archaeological study using a small number of sonar scans taken with a low-payload micro-ROV. In addition, the work has demonstrated a method of affixing depth data captured in low cost stereo cameras to enhance rough sonar generated reconstructions. Previous work has successfully created 2D and extruded 2.5D models of closed underwater systems, while the work here presents an initial success for a unified solution for surface reconstruction from 3D sonar scans with stereo image enhancements. We were able to successfully build representational surface reconstructions given sparse 3D sonar data and integrate locally higher resolution stereo data to add geometric details when possible.

Three large sonar data sets were reconstructed to test the proposed pipeline's ability to handle sparse 3D sonar data. Two of the three data sets contained only horizontal scans taken incrementally along the depth of the site, and one data set was collected using both a vertical and horizontal sonar on the ROV. All three of the reconstructed models are visually appealing, representative of true data, scaled according to ground truth data, and useful for archaeological study. The process of producing accurate disparity maps from left and right images proved difficult underwater, hampering the virtues of the second phase of the pipeline. However, initial results have validated the potential to incorporate higher resolution stereo image data with the coarse resolution sonar data.

Several stages of the pipeline could be improved in future work. An octree could be substituted for the volume data structure in order to prevent memory limitations when subdividing voxels, replacing marching cubes with a dual contouring method $(\mathrm{Ju}$ et al., 2002) to prevent cracks and integrating multiresolution solutions for addressing the difference in data density between stereo and sonar data. Projectors could be automatically aligned in the volume using visual SLAM, or could have their positions determined before runtime using a localization device on the ROV such as an IMU or a SmartTether. With regards to stereo hardware, the proposed stereo algorithm would greatly benefit from cameras with a wider baseline and lenses corrected for underwater photography. Additionally, a more uniform structured lighting system would be useful in illuminating the scene properly.

\section{ACKNOWLEDGEMENTS}

We would especially like to thank Dr. Jane Lehr and 2013 ICEX teams for their contributions. This ma- 
terial is based upon work supported by the National Science Foundation under Grant No. 0966608.

\section{REFERENCES}

Adalsteinsson, D. and Sethian, J. A. (1994). A fast level set method for propagating interfaces. Journal of Computational Physics, pages 269-277.

Beall, C., Lawrence, B. J., Ila, V., and Dellaert, F. (2010). 3d reconstruction of underwater structures. In Intelligent Robots and Systems (IROS), 2010 IEEE/RSJ International Conference on, pages 4418-4423. IEEE.

Bresenham, J. E. (1965). Algorithm for computer control of a digital plotter. IBM Systems journal, 4(1):25-30.

Campos, R., Garcia, R., and Nicosevici, T. (2011). Surface reconstruction methods for the recovery of $3 \mathrm{~d}$ models from underwater interest areas. In OCEANS, 2011 IEEE - Spain, pages 1-10.

Deng, S., Li, Y., Jiang, L., Cao, Y., and Zhang, J. (2011). Variational surface reconstruction from sparse and nonparallel contours for freehand $3 \mathrm{~d}$ ultrasound. In Informatics in Control, Automation and Robotics, pages 51-58. Springer.

Dobke, A., Vasquez, J., Lieu, L., Chasnov, B., Clark, C., Dunn, I., Wood, Z., and Timothy, G. (2013). Towards three-dimensional underwater mapping without odometry. In To appear in: Proceedings of the 18th International Symposium on Unmanned Untethered Submersible Technology (UUST).

Drap, P., Seinturier, J., Scaradozzi, D., Gambogi, P., Long, L., and Gauch, F. (2007). Photogrammetry for virtual exploration of underwater archeological sites. In Proceedings of the 21st International Symposium, CIPA 2007: AntiCIPAting the Future of the Cultural Past: Athens (Greece), 01-06 October 2007. Citeseer.

Forney, C., Forrester, J., Bagley, B., McVicker, W., White, J., Smith, T., Batryn, J., Gonzalez, A., Lehr, J., Gambin, T., et al. (2011). Surface reconstruction of maltese cisterns using rov sonar data for archeological study. In Advances in Visual Computing, pages 461471. Springer.

Forrester, J., McVicker, W., Gambin, T., Clark, C., and Wood, Z. J. (2013). Uncertainty visualization and hole filling for geometric models of ancient water system. In Proceedings of the 4th International Conference on Information Visualization Theory and Application (IVAPP).

Hurtós, M., i Soler, X. C., and Salvi, J. (2009). Integration of optical and acoustic sensors for $\mathrm{d}$ underwater scene reconstruction. Instrumentation viewpoint, (8):43.

Ju, T., Losasso, F., Schaefer, S., and Warren, J. (2002). Dual contouring of hermite data. ACM Trans. Graph., 21(3):339-346.

Kazhdan, M., Bolitho, M., and Hoppe, H. (2006). Poisson surface reconstruction. In Proceedings of the fourth Eurographics symposium on Geometry processing.

Lorensen, W. E. and Cline, H. E. (1987). Marching cubes: A high resolution $3 \mathrm{~d}$ surface construction algorithm.
In ACM Siggraph Computer Graphics, volume 21, pages 163-169. ACM.

Mahon, I., Pizarro, O., Johnson-Roberson, M., Friedman, A., Williams, S. B., and Henderson, J. C. (2011). Reconstructing pavlopetri: Mapping the world's oldest submerged town using stereo-vision. In Robotics and Automation (ICRA), 2011 IEEE International Conference on, pages 2315-2321. IEEE.

McVicker, W., Forrester, J., Gambin, T., Lehr, J., Wood, Z. J., and Clark, C. M. (2012). Mapping and visualizing ancient water storage systems with an rov an approach based on fusing stationary scans within a particle filter. In Robotics and Biomimetics (ROBIO), 2012 IEEE International Conference on, pages 538544. IEEE.

Mullen, P., De Goes, F., Desbrun, M., Cohen-Steiner, D., and Alliez, P. (2010). Signing the unsigned: Robust surface reconstruction from raw pointsets. In Computer Graphics Forum, volume 29, pages 1733-1741. Wiley Online Library.

Nalpantidis, L. and Gasteratos, A. (2010). Stereo vision for robotic applications in the presence of non-ideal lighting conditions. Image and Vision Computing, 28(6):940-951.

Nascimento, E., Campos, M., and Barros, W. (2009). Stereo based structure recovery of underwater scenes from automatically restored images. In Computer Graphics and Image Processing (SIBGRAPI), 2009 XXII Brazilian Symposium on, pages 330-337. IEEE.

Osher, S. and Fedkiw, R. (2003). Level set methods and dynamic implicit surfaces, volume 153. Springer Verlag.

Papaleo, L. and Puppo, E. (2004). Online Data Fusion for building 3D models from acoustical range. Technical Report DISI-TR-05-04, University of Genov.

Scharstein, D. and Szeliski, R. (1998). Stereo matching with nonlinear diffusion. International Journal of Computer Vision, 28:155-174. 10.1023/A: 1008015117424.

Schmidt, V. E. and Rzhanov, Y. (2012). Measurement of micro-bathymetry with a gopro underwater stereo camera pair. In Oceans, 2012, pages 1-6. IEEE.

Sethian, J. (2001). Evolution, implementation, and application of level set and fast marching methods for advancing fronts. Journal of Computational Physics, 169(2):503-555.

Swirski, Y., Schechner, Y. Y., Herzberg, B., and Negahdaripour, S. (2010). Underwater stereo using natural flickering illumination. In OCEANS 2010, pages 1-7. IEEE.

Zhao, H.-K., Osher, S., and Fedkiw, R. (2001). Fast surface reconstruction using the level set method. In Variational and Level Set Methods in Computer Vision, 2001. Proceedings. IEEE Workshop on, pages 194201. IEEE.

Zitnick, C. L. and Kanade, T. (2000). A cooperative algorithm for stereo matching and occlusion detection. Pattern Analysis and Machine Intelligence, IEEE Transactions on, 22(7):675-684. 